\title{
A Model-Based Integrated Approach to Track Myocardial Deformation Using Displacement and Velocity Constraints
}

\author{
Pengcheng Shi*, Glynn Robinson ${ }^{\dagger}$, R. Todd Constable ${ }^{\dagger}$, Albert Sinusas ${ }^{\dagger} \ddagger$, and James Duncan ${ }^{\dagger, *}$ \\ Image Processing and Analysis Group \\ Departments of *Electrical Engineering, ${ }^{\dagger}$ Diagnostic Radiology, and $\ddagger_{\text {Medicine }}$ \\ Yale University, New Haven, CT 06520-8042 \\ xship@noodle.med.yale.edu
}

\begin{abstract}
Accurate estimation of heart wall dense field motion and deformation could help to better understand the physiological processes associated with ischemic heart diseases, and to provide significant improvement in patient treatment. We present a new method of estimating left ventricular deformation which integrates instantaneous velocity information obtained within the mid-wall region with shape information found on the boundaries of the left ventricle. Velocity information is obtained from phase contrast magnetic resonance images, and boundary information is obtained from shape-based motion tracking of the endo- and epicardial boundaries. The integration takes place within a continuum biomechanical heart model which is embedded in a finite element framework. We also employ a feedback mechanism to improve tracking accuracy. The integration of the two disparate but complementary sources overcomes some of the limitations of previous work in the field which concentrates on motion estimation from a single image-derived source.
\end{abstract}

\section{Introduction}

The measurement of regional myocardial injury due to ischemic heart diseases is an important clinical problem. Accurate estimates of heart motion and deformation are essential to evaluate normal and abnormal cardiac physiology and mechanics. It is the goal of many forms of cardiac imaging and image analysis methods to measure the regional function of the left ventricle (LV) in an effort to isolate the location, severity and extent of ischemic myocardium. The complexity of the LV motion and the absence of internal landmarks in the myocardium imply that the true motion trajectories of tissue elements are, at best, difficult to infer from sequential images.

Recently, the advances in magnetic resonance (MR) imaging technology have provided new possible strategies to the quantification of the LV motion and function. One technique has been the MR tagging of the myocardium $[1,18]$, which creates a sparse magnetization grid that tags the underlying tissue, and uses the grid deformation to follow the tissue movement. However, the magnetic grid tends to decay over time, which limits the its ability to track motion over the entire cardiac cycle. It is also quite difficult to acquire and assemble the detected tags into a robust 3D anal- ysis framework. We note, however, that the efforts of Young [17] and Prince[5] have developed approaches for assembling MR tagging-derived information into three dimensional maps of cardiac motion/function. Another new approach for point tracking is the use of phase contrast images to decipher local velocity which in turn can be integrated in order to estimate trajectories of individual points over time[4]. Currently, phase contrast velocity estimates near the endo- and epicardial boundaries are extremely noisy because they include information outside the myocardium. Thus, as with MR tagging, the most accurate LV motion information is obtained from the middle of the myocardial wall, and is least reliable near the wall boundaries. Nevertheless, the mid-wall instantaneous velocity provides valuable information, and we intend to use it as one source of constraints in our integrated framework.

In computer vision research, nonrigid surface registration and nonrigid motion tracking are directly relevant to the cardiac motion problem. The efforts in nonrigid surface registration attempt to register entire sets of image data and quantitatively and statistically look for similarities and differences, which include the use of deformable thin-plate splines[3] and octreespline based volumetric transformation[14]. The more physical-model motivated work of Pentland[7] and Terzopolous[15] is also aimed at solving this embedding problem. In these approaches, any estimates of correspondence between points are either specifically assumed to be known to aid in solving the problem, or are not considered at all. The goal of the nonrigid motion recovery is to find point correspondences between two objects over as dense a spatial field as possible. Goldgof $[9]$ has been pursuing surface shape matching ideas using conformal stretching model. Recently, Metaxas[11] has utilized the mid-wall point correspondences estimated from MR tagging in conjunction with the deformable superquadrics.

We have proposed a shape-based approach to compute point-wise myocardial boundary (contour and surface) motion between successive image frames $[6$, 13]. Our method has been based on locating and matching boundary geometric landmarks and using a mathematical optimization reasoning strategy to combine the data-derived information with a local coherent smoothness model. However, we now believe 
that solely boundary based descriptions as well as the purely mid-wall based methods are inherently incomplete, with each neglecting valuable additional constraints provided by the other. Therefore, we propose here an integrated motion tracking framework that combines the boundary (from our shape based approach) and mid-wall (from phase contrast MR images) information under a continuum mechanical model of the LV. This heart model enables us to naturally incorporate actual physical constraints related to known cardiac parameters, such as pressures, elasticity, etc. We also employ a feedback mechanism which minimizes the differences between the local real data boundary and predicted boundary to correct the boundary matching error and improve the accuracy of the motion tracking. The proposed approach makes it possible to more reliably assess the myocardial deformation within the entire left ventricle. We want to emphasize that although we adopt the finite element framework as in some previous work, our underlying model is based on actual physical constraints of the myocardium, and we are considering the whole myocardium (epicardium, endocardium, and mid-wall) as one single object, and integrating of complementary information from boundary and mid-wall, while others only consider either boundary or mid-wall but not both. In addition, unlike other authors, our goal is to find point correspondence between image frames.

In the rest of the paper, analysis will be given in the two dimensional case. However, the theoretical aspect of the approach is also valid in 3D, though it may be more constrained computationally. We are currently implementing one version of the 3D approach.

\section{Image-Derived Information}

In this section, we briefly describe the two sources of image-derived information, the boundary displacement and the mid-wall instantaneous velocity. See the cited references for more details.

\subsection{Shape-Based Boundary Displacement}

As mentioned above, we have previously proposed that we can use the shape of the endo- and epi-cardial boundary to track the motion of a dense field of points which sample the boundary over the entire cardiac cycle[6, 13]. Our motion tracking method is based on locating and matching differential geometric landmarks and using a mathematical optimization reasoning strategy to combine a local coherent smoothness model with data-derived information to obtain dense field motion vectors. In the $2 \mathrm{D}$ case, a sparse subset of the contour points are created by choosing shape landmarks which are defined as locally extreme curvature points or curvature zero-crossing points. Making the curvature zero-crossing points part of the landmark subset ensures that any contour segment between two consecutive landmarks will not change curvature sign if we traverse the segment from one end to the other. In other words, all the contour segments constructed by the consecutive landmarks will be either constant convex, or constant concave, or a straight line. The significance of this classification will be explained in the feedback mechanism section.

Computation of the displacement vector flow field is carried out in two sequential steps for a given pair of contours representing two consecutive time frames: computation of initial local matching of landmarks of the contours, and formulation/solution of a regularization functional whose solution results in a set of displacement vectors which are locally smooth but still adhere to the initial matching. The bending energy matching criterion is defined as $[6]$ :

$$
e_{\text {bend }}(\delta)=\frac{1}{2} E I \int_{\mathcal{C}}\left[\kappa_{g}(\tau+\delta)-\kappa_{f}(\tau)\right]^{2} d \tau
$$

where $\kappa_{f}$ is the curvature for any given landmark point in the first contour, $\mathcal{C}$ the search region on the second contour, $\kappa_{g}$ the curvature of a candidate point within the search region on the second contour, and $\delta$ indexes the different candidate points within search region. Among all the candidate points within the search region, the one which yields the smallest bending energy $e_{\text {bend }}$ is chosen as the initially matched point. The result of this process for every landmark produces a set of shape-based, best-matched initial motion vectors $\mathbf{U}_{\text {init }}(t)$ for each pair of contours. Next, for each contour pair, a regularization procedure is adopted to result in a dense velocity vector field $\mathbf{U}_{\text {optimal }}(t)$ that is an optimal compromise between an adherence term and a smoothness term. A subset of optimal vectors, denoted as $\mathbf{U}_{\text {boundary }}(t)$, which is comprised of all the vectors that terminate at the landmarks of the second contour is then formed. We will use this subset of boundary displacement vectors as the shape-derived boundary information in our integrated framework.

\subsection{Phase Contrast Images and Mid-Wall Instantaneous Velocity}

Recently, a new imaging technique[12], Phase Velocity Magnetic Resonance Imaging, has been developed. It incorporates velocity phase encoding into a conventional cine-MR sequence and produces images of myocardial instantaneous velocity throughout the cardiac cycle. Phase contrast methods can depict motion parameters with pixel precision and thus provide a high resolution technique for quantitative measurements. Led by Dr. Constable, our team has been working on developing methods to deal with propagated phase errors, as well as quantitatively characterizing the phase velocity based motion tracking[4].

Uniform motion of tissue in the presence of a magnetic field gradient produces a change in the MR signal phase, $\phi$, proportional to the velocity:

$$
\phi=\nu \gamma \int_{0}^{T E} G(t) t d t
$$

where $G(t)$ is the gradient strength as a function of time, $\nu$ the tissue velocity, $T E$ the echo delay time, and $\gamma$ the gyromagnetic ratio. The velocity in a particular spatial direction can be estimated by measuring the difference in phase shift between two acquisitions with different first gradient moments. Velocity maps encoded for motion in three spatial dimensions may easily be obtained at multiple time instances throughout the cardiac cycle using a phase contrast cine-MR imaging sequence. The acquired velocity maps may then be used to provide information on tissue displacement, strain, strain rate, and other quantitative measures of deformation. Regardless of which quantitative parameters are to be measured, an important first 
step is to devise methods that can accurately track each segment of myocardium as it deforms through the heart cycle. However, the velocity maps themselves only provide instantaneous motion information. They do not establish the point correspondences between image frames. Methods that use direct forward and/or backward integration of velocity to estimate the displacement vector have been proposed[4]. These methods assume various kinds of constant velocity conditions between time frames. These assumptions suffer from the fact that myocardial motion is the result of complex interaction between electrical activation, myocardium active contraction, blood flow pressure, etc, and it is not constant between even small time intervals. Errors resulting from the constant velocity assumptions can be significant especially during the fast contraction stage, which is very important in the prediction of cardiac health.

As mentioned before, phase contrast velocities near the endo- and epi-cardial boundaries are extremely noisy, reliable motion information is only available within the mid-wall region. We denote these mid-wall velocity vectors as $\dot{\mathbf{U}}_{\text {mid-wall }}(t)$.

\section{A Model Based Integrated Approach with Feedback Mechanism}

In this section, we present a new method of estimating left ventricular deformation which integrates mid-wall instantaneous phase contrast velocity with boundary shape-based displacement. ${ }^{1}$ The integration takes place within a continuum mechanical model of the LV, is embedded in a finite element framework, and uses a feedback mechanism to improve accuracy. The integration of the two disparate but complementary sources overcomes the limitations of previous work in the field which concentrates on motion estimation from a single image-derived source.

\subsection{Continuum Mechanical Model of the Left Ventricle}

We want to model the left ventricle such that given image-derived constraints and other prescribed conditions, the model should provide a realistic and reasonable framework to help recover nonrigid deformation. In the work proposed here, we represent each imaged myocardial slice as a homogeneous elastic solid continuum, bounded by endo- and epi-cardial contours. We want to emphasize that this model is different from the spring-like mesh widely used in graphics and vision community, it is a model based on continuum mechanics. Since we intend to embed our model in a finite element framework, and use the principle of minimum potential energy to solve the governing equilibrium equations for motion recovery, we need to establish the relationship between the deformation and the induced stress. Rigid motion induces no stress, thus the displacements of each point of the body cause no strain. The heart is a nonrigid object that deforms over time and has very complicated biomechanical

\footnotetext{
${ }^{1}$ The regular MR images on which we perform our boundary based method and the phase contrast images from which we derive the velocity information are the magnitude and phase images of the same data acquisition, respectively. Spatially, they are perfectly registered with each other.
}

properties $[8,10]$ and stress-strain relationships. For the simplicity of the framework, we assume that the myocardium is a Hookean material, with its $\operatorname{stress}([\sigma])$ and $\operatorname{strain}([\varepsilon])$ relationship (the constitutive equation of the material) obeying Hooke's law: ${ }^{2}$

$$
[\sigma]=[D][\varepsilon]
$$

Under two dimensional Cartesian coordinate system, assuming the displacement along the $\mathrm{x}$ - and $\mathrm{y}$-axis of a point to be $u(x, y)$ and $v(x, y)$ respectively, the strain tensor $[\varepsilon]$ of the point can be expressed as:

$$
[\varepsilon]=\left[\begin{array}{c}
\frac{\partial u}{\partial x} \\
\frac{\partial v}{\partial y} \\
\frac{\partial u}{\partial y}+\frac{\partial v}{\partial x}
\end{array}\right]
$$

and under plane strain condition, matrix $D$ can be derived to be:

$$
[D]=\frac{E}{(1+\nu)(1-2 \nu)}\left[\begin{array}{clc}
1-\nu & \nu & 0 \\
\nu & 1-\nu & 0 \\
0 & 0 & \frac{1-2 \nu}{2}
\end{array}\right]
$$

Here, E and $\nu$, Young's modulus and Poisson's ratio, are two material-related constants which have been established experimentally for myocardium in biomechanics literature[16], with value to be around 75,000 Pascal and 0.5 respectively.

It is clear that under our model, the internal stress caused by the deformation is a function of the displacement vector and some material-specific constants. We are going to use this strain-stress relationship in the finite element representation of the model.

\subsection{Finite Element Representation of the Model}

The finite element method is an engineering mechanics technique for analyzing the dynamic behavior of an object. The first step in this method is to divide the continuous structure of the object into finite pieces, or elements, and to construct a finite element mesh to represent the object. Since the velocity constraint is only reliable in the mid-wall region, we want to incorporate as many mid-wall elements as possible. Currently, pixel-resolution grids are used for 2D applications. ${ }^{3}$ The Delaunay tessellation method is used to tessellate the bounded LV into a triangular grid. Next, local basis functions for each element are constructed. These allow approximation of the properties of any point by those of the nodes of the element to which it belongs. An isoparametric formulation defined in a natural coordinate system is used, in which the interpolation of the element coordinates and element displacements use the same basis functions. For the tri-nodal linear element, the basis functions are linear functions of the three nodes' coordinates[2].

\footnotetext{
${ }^{2}$ However, currently we are in the process of developing more suitable and realistic constitutive models, any of which can be inserted into this framework.

${ }^{3}$ However, we are investigating methods to reduce the resolution in the $3 \mathrm{D}$ case for computational reasons.
} 

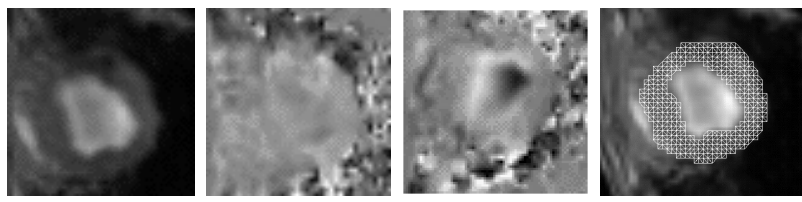

Figure 1: Regular magnitude MR image, x-direction phase contrast image, $y$-direction phase contrast image, and triangular grid superimposed on the region of interest.

The nodal displacement based governing dynamic equation of each element is established under the principle of minimum potential energy, using the mechanical model described in the previous section. The equations are assembled together in matrix form as:

$$
\mathbf{M U ̈}+\mathbf{C U}+\mathbf{K U}=\mathbf{R}
$$

where $\mathbf{M}$ is the mass matrix, $\mathbf{C}$ the damping matrix, $\mathbf{K}$ the stiffness matrix, $\mathbf{R}$ the force load, and $\mathbf{U}$ the nodal displacement vector field. $\mathbf{M}$ is a function of the element basis functions and material density $\left(1.1 \mathrm{gram} / \mathrm{mm}^{3}\right)$, and $\mathbf{K}$ is a function of element basis functions and material strain-stress matrix $[D]$. Meanwhile, matrix $\mathbf{C}$ is frequency dependent, and is proportional to the mass matrix. Considering the motion characteristics of the heart tissue (a very low damping system), we choose element of $\mathbf{C}$ to be about one percent of the value of the corresponding element of M. We want to point out that we also intend to use this model to enforce certain real physical constraints related to known cardiac pressures.

It is important to note that while the finite element grid provides the basis for approximating a continuous spatial model, the dynamic equations provide the basis of an appropriate temporal model for the matching and predicting of image frames.

\subsection{Numerical Solution of the System}

The governing equations have been constructed in such a way that the mid-wall instantaneous velocity and the boundary displacement are being used as data-based constraints within a continuum mechanical model. Dense deformation fields, (displacement, velocity, acceleration, and strain), can be estimated from this model-based, image-constrained framework.

For any sampled time instant (assume $t=T_{0}$ for convenience), the initial conditions for the system of differential equations are as follows:

- The initial displacements of all the points $\mathbf{U}_{\text {all }}\left(t_{0}\right)$ are always zero (the object has not moved yet).

- The phase images provide the initial velocity information $\dot{\mathbf{U}}_{\text {mid-wall }}\left(t_{0}\right)$ of the mid-wall points. For the other points, we could assume $\dot{\mathbf{U}}_{\text {other }}\left(t_{0}\right)$ to be zero. However, if we take into account the periodic nature of the heart motion, we can use the estimated velocity at these points from the solution of equations at the previous time instant as the initial velocity at this time.

- The initial acceleration of all the points $\ddot{\mathbf{U}}_{a l l}\left(t_{0}\right)$ could be zero, or we can again use the estimated result from the previous time.

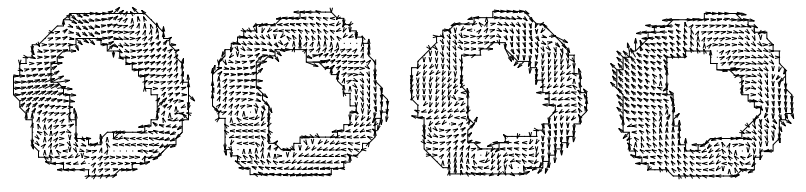

Figure 2: The dense field displacement vector maps of four consecutive time frames, beginning with the ED frame. Note the non-homogeneous nature of myocardial motion.

- The initial equivalent total load $\mathbf{R}\left(t_{0}\right)$ can be computed from the governing equations:

$$
\mathbf{R}\left(\mathbf{t}_{\mathbf{0}}\right)=\mathbf{M} \ddot{\mathbf{U}}\left(\mathbf{t}_{\mathbf{0}}\right)+\mathbf{C} \dot{\mathbf{U}}\left(\mathbf{t}_{\mathbf{0}}\right)
$$

where $\dot{\mathbf{U}}\left(t_{0}\right)$ and $\ddot{\mathbf{U}}\left(t_{0}\right)$ are known.

We also want to enforce the displacements of the boundary landmarks $\mathbf{U}_{\text {boundary }}\left(t_{1}\right)$ when the myocardium deforms into the next time instant $t_{1}$. One method to incorporate this constraint is as follows: assume that the $i^{\text {th }}$ component of vector $\mathbf{U}$ has enforced value $b$, we multiply the $i i^{t h}$ element of the stiffness matrix $\mathbf{K}$ by $N$, and replace the $i^{\text {th }}$ element of the load vector $\mathbf{R}$ by $N b$, where $N$ is a very large number. Following this manipulation, the modified governing equations must now give $U_{i}\left(t_{1}\right)=b$.

Through the construction of the initial conditions, and the enforcement of boundary landmarks displacements, the solution to the governing equations will be the result of the integration of two sources of imagederived information under the guidance of the mechanical model. The step-by-step solution of the system is performed using the Newmark integration method, which is unconditionally stable. The integration process results in estimates of displacement of all the node points between time intervals, as well as the predicted nodal velocity and acceleration at the next time instant.

\subsection{Feedback Mechanism to Improve Ac- curacy}

When using the finite element framework to track motion between temporal images, it can be regarded as a predictor from time $t_{0}$ to time $t_{1}$. Since we already have data information at $t_{1}$ in terms of the segmented endo- and epi-cardial contours, as well as the mid-wall velocity, we can refine the mapping estimate between frames $t_{0}$ and $t_{1}$ by comparing how close the image-driven, model-based prediction comes to the actual data. The differences between the data and the prediction can be used as a recursive feedback term to improve the matching process. Here, we report our progress in using the boundary difference as the feedback force.

As described in section 2, the boundary displacement constraint is only enforced at the matching vectors which terminate at the landmark points of the second contour. This way, the real and predicted contours at time $t_{1}$ intersect at these points. As explained before, the contour segments defined by two consecutive landmarks of the real data will always be either constant convex, or constant concave, or a straight line. The corresponding predicted contour segments 


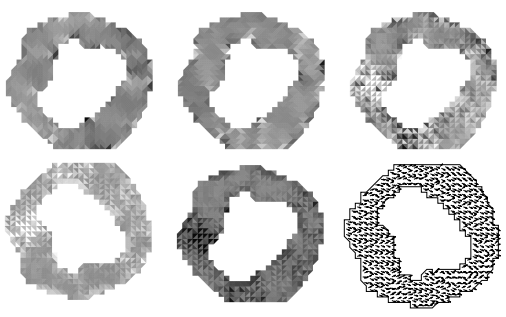

Figure 3: Top: coordinate-dependent strain maps: $\mathrm{x}$ direction, y direction, and shear strain; Bottom: principal strain maps: maximum strain, minimum strain, and the direction associated with the maximum principal strain.

should be close to real ones in the same classification sense, if the initial shape-based boundary matching is reasonably good and the contour segments are not too large. In practice, these two requirements are readily satisfied. In the feedback process, predicted contour segments are compared to actual ones, based on a metric related to the similarity between pairs of actual and predicted contour segments. To date, two curve comparison metrics have been developed, and have shown similar effects in improving the matching process.

The first metric utilizes the shape matching idea that our initial boundary matching is based upon. However, we were using point-wise shape comparison in that process, but now we have to compare curve segments. We define that the total tension for any curve segment $\mathcal{C}$ equal to its normalized total curvature:

$$
T(\mathcal{C})=\int_{\mathcal{C}}|\kappa(s)| d s
$$

Since we have stated that the real and predicted contour segments would be close in the sense of constant convexity or concavity, the total tension of any curve segment will indicate its total curveness. Furthermore, since the whole real contour can be treated as if it were in equilibrium state tension-wise, the total tension difference (termed as expanding force $f(\mathcal{P})$ ) between the corresponding predicted segment $\mathcal{P}$ and real segment $\mathcal{R}$ can be used as a target as to how much the predicted segment should increase or decrease its tension:

$$
f(\mathcal{P})=T(\mathcal{P})-T(\mathcal{R})
$$

Positive $f(\mathcal{P})$ indicates that the segment has a tendency to expand along the outward tangent directions of the two end points, while negative $f(\mathcal{P})$ implies the shrinking tendency. Since the neighboring segments interact at the common end point, the net expanding force of the two segments determines the net expanding/shrinking tendency of the common landmarks.

The second comparison metric simply defines the total tension for any given curve segment equal to the normalized area of the segment (closed by the line connecting the two end points). The expanding force is again defined as the total tension difference between the corresponding predicted and real segments.

The right figure of Fig. 4 illustrates the contour segment comparison idea described above. Here, the solid line represents the real contour, and the dotted

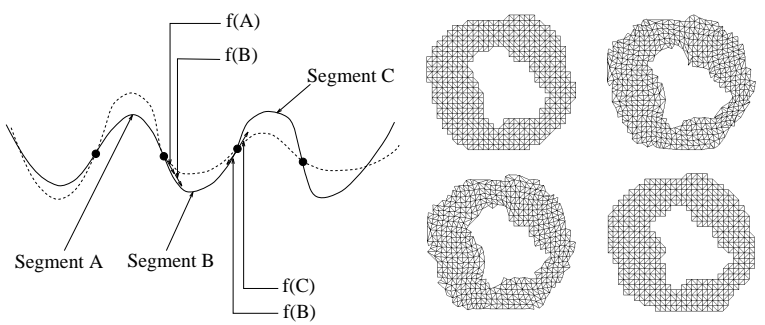

Figure 4: Left: compute the landmark net expanding/shrinking force in the feedback mechanism. Right: top left: original grid; top right: predicted grid without feedback; bottom left: predicted grid with two feedback iterations; bottom right: actual target grid.

line is the predicted contour. Segments $A$ and $C$ have expanding tendency, while segment $B$ is inclined to shrink. However, the net force at the common end of $A$ and $B$ causes that landmark expand (or shrink from $B$ 's standpoint) into $B$, while the net force at the common end of $B$ and $C$ is minimal (no change).

After forming the net expanding force at each landmark point, adjustments are made for the initial boundary displacements. Displacement vectors emanating from the first image frame are modified so they will end on the boundary of the second image, at one side or another of the original matching landmarks, depending on the magnitude and direction of the net expending force of each landmark. The new displacement is then applied into the integrated finite element framework to compute refined motion information.

\section{Experimental Results}

The model-based integrated framework proposed in this paper has been implemented for the two dimensional case. Experiments have been conducted with real cardiac phase contrast images from a canine study.

Figure 1 demonstrates the image dataset we are currently working with. Shown here are three MR images taken at the end diastolic (ED) stages of the cardiac cycle (we have sixteen time frames within each cycle): the regular magnitude MR image which provides the anatomical information of the left ventricle, the $\mathrm{x}$ and $y$-direction phase contrast images which indicate the instantaneous velocity components in the respective directions. The right figure shows the triangular finite element grid representation of a 2D myocardial slice (superimposed on the region of interest).

Figure 2 presents the dense field displacement vector maps of four consecutive time frames, beginning with the ED frame. Here, a vector arrow begins from its position at present time $t$, ends at its position of next time $t+\delta t$. The non-homogeneous nature of myocardial motion is very evident from these displacement maps: within the same time frame, different regions of the myocardium display very different motion characteristics (direction, magnitude, etc); between time frames, the same muscle tissue changes its motion parameters within a fairly short time interval.

We believe more detailed and sophisticated analysis is required to infer more useful information for clinical and research purposes. To that end, we have calculated the strain tensors for each triangular elements of the finite element grid, which indicates the 
non-rigid deformation but not the rigid bulk motion of the element. The top row of Figure 3 shows the three components of the strain tensors: $\mathrm{x}$ component, y component, and shear strain. The bottom row of the figure shows the more object centered principal strain maps: maximum, minimum, shear, and the principal direction associated with the maximum principal normal strain. In our original color-coded display of the strain maps, different colors are used to delineate the positive and negative values of the strain components. The gray scale maps presented here can be difficult to interpret. We denote large positive strain with light white shade, large negative strain with dark shade, and near zero strain with neutral gray. These strain maps display the evidence of the non-homogeneous deformation.

Finally, the left figure of Fig. 4 illustrates the mechanism behind the adjustments of the boundary displacements. The right figures show the results of feedback mechanism to improve the accuracy of the matching process. Top left is the original grid, bottom right is the target grid at the next time frame. Top right is the system predicted grid without any corrections, while bottom left is the refined prediction after two steps of feedback correction at the boundaries. We can see the local and overall improvement of the refined prediction.

\section{Conclusions}

We have presented an integrated motion tracking framework that combines the boundary displacement and mid-wall instantaneous velocity information under a continuum mechanical model of the left ventricle. The heart model naturally enables us to incorporate actual physical constraints related to known cardiac parameters and myocardium properties. We also employ a feedback mechanism which minimizes the differences between the local real data boundary and predicted boundary to correct the boundary matching error and improve the accuracy of the motion tracking. We believe our approach is one of the first efforts to integrate disparate but complementary image-derived modules to assess the true transmural myocardial deformation. Initial results from the analysis of real images have been encouraging. And we are moving towards developing 3D framework.

\section{References}

[1] L. Axel and L. Dougherty. Mr imaging of motion with spatial modulation of magnetization. Radiology, 171:841-845, 1989.

[2] K. Bathe and E. Wilson. Numerical Methods in Finite Element Analysis. Prentice-Hall, New Jersey, 1976.

[3] F. L. Bookstein. Principal warps: Thin-plate splines and the decomposition of deformations. IEEE Trans. on Patt. Anal. and Mach. Intell., pages $567-585,1989$.

[4] R.T. Constable, K. Rath, A. Sinusas, and J. Gore. Development and evaluation of tracking algorithms for cardiac wall motion analysis using phase velocity $\mathrm{mr}$ imaging. Magnetic Resonance in Medicine, 32:33-42, 1994.
[5] T. S. Denney and J. L. Prince. 3d displacement field reconstruction from planar tagged cardiac $\mathrm{mr}$ images. In IEEE Workshop on Biomedical Image Analysis, pages 51-60, 1994.

[6] J. S. Duncan, R. Owen, L. Staib, and P. Anandan. Measurement of non-rigid motion using contour shape descriptors. In IEEE Conf. on CVPR, pages 318-324, Maui, June 1991.

[7] B. Horowitz and S. Pentland. Recovery of nonrigid motion and structure. In IEEE Conf. on CVPR, pages 325-330, Maui, June 1991.

[8] P. J. Hunter, P. M. F. Nielsen, B. H. Smaill, and I. J. LeGrice. An anatomical heart model with applications to myocardial activation and ventricular mechanics. Critical Reviews in Biomedical Engineering, 20:403-426, 1992.

[9] C. Kambhamettu and D. Goldgof. Point correspondence recovery in non-rigid motion. In IEEE Conf. on CVPR, pages 222-227, June 1992.

[10] A. McCulloch and J. Omens. Non-homogeneous analysis of three-dimensional transmural finite deformation in canine ventricular myocardium. $J$. Biomechanics, 24:539-548, 1991.

[11] J. Park, D. Metaxas, and A. Young. Deformable models with parameter functions: Application to heart wall modeling. In IEEE Conf. on $C V P R$, pages 437-442, June 1993.

[12] N. J. Pelc, A. Shimakawa, and G. H. Glover. Phase contrast cine MRI. In Proc. of the 8th Annual SMRM, page 101, Amsterdam, 1989.

[13] P. Shi, A. Amini, G. Robinson, A. Sinusas, C. T. Constable, and J. Duncan. Shape-based 4d left ventricular myocardial function analysis. In $I E E E$ Workshop on Biomedical Image Analysis, pages 88-97, 1994.

[14] R. Szeliski and S. Lavallee. Matching 3d anatomical surfaces with non-rigid deformations using octree-splines. In IEEE Workshop on Biomedical Image Analysis, pages 144-153, 1994.

[15] D. Terzopolous and D. Metaxas. Dynamic 3d models with local and global deformation: Deformable superquadrics. IEEE Trans. on Patt. Anal. and Mach. Intell., 13(17), 1991.

[16] H. Yamada. Strength of Biological Material. The Williams and Wilkins Company, Baltimore, 1970.

[17] A. A. Young, D. L. Kraitchman, and L. Axel. Deformable models for tagged mr images: Reconstruction of two- and three-dimensional heart wall motion. In IEEE Workshop on Biomedical Image Analysis, pages 317-323, 1994.

[18] E. Zerhouni and et. al. Tagging of the human heart by multiplanar selective RF saturation for the analysis of myocardial contraction. In $A b$ stracts of the Ann. Meeting of the Soc. of MR in Imaging, page 10, San Francisco, 1988. 